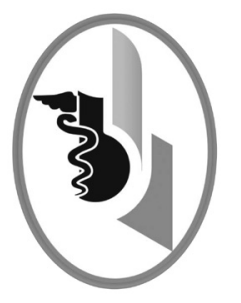

\title{
PREVALENCIA DE DISFUNCIÓN SEXUAL EN MUJERES DEL EJE CAFETERO
}

\author{
PREVALENCE OF SEXUALDYSFUNCTION IN WOMEN FROM \\ EJE CAFETERO-COLOMBIA
}

Espitia-De La Hoz Franklin José ${ }^{1}$

Correspondencia: espitiafranklin@hotmail.com

Recibido para evaluación: agosto 05 de 2016. Aceptado para publicación: marzo 05 de 2016.

\section{RESUMEN}

Introducción: las disfunciones sexuales son alteraciones en las fases del ciclo de respuesta sexual que impiden o dificultan disfrutar satisfactoriamente de la sexualidad. Las mujeres pueden presentar trastornos del deseo, de la excitación, del orgasmo o dolor, aunque es frecuente la coexistencia de dos o más disfunciones.

Objetivo: determinar la prevalencia de disfunción sexual femenina en una población del Eje Cafetero colombiano.

Materiales y métodos: estudio prospectivo, trasversal, descriptivo y aplicativo. Se aplicó y se midió el Índice de Función Sexual Femenina (IFSF) en mujeres de 18 a 84 años de edad que consultaron seis de los principales centros de salud de las ciudades del Eje Cafetero (Armenia, Manizales y Pereira).

Resultados: respecto a la prevalencia de las diferentes disfunciones sexuales, en la población general, la más frecuente fue el trastorno del deseo sexual (34.9\%), seguida de ausencia o infrecuencia en el orgasmo (28.5\%), falta de lubricación, trastorno de la excitación (20.2\%) y dolor en la relación sexual (8.4\%). Las mujeres en edad reproductiva mostraron prevalencia del $27.4 \%$ de trastornos del deseo, mientras que en las mujeres en etapa menopáusica mostraron prevalencia del $42.9 \%$ de trastornos del deseo. La disfunción del deseo sexual se asoció a menor satisfacción en la relación sexual y de pareja con estados emocionales negativos $(p<0.001)$.

Conclusión: existe una proporción importante de mujeres con disfunción sexual (34.9\%) en el Eje Cafetero colombiano. Rev.cienc.biomed. 2016;7(1):25-33.

\section{PALABRAS CLAVE:}

Disfunción sexual; Epidemiología; Hábitos sexuales.

\section{SUMMARY}

Introduction: the sexual dysfunctions are alterations in the phases of sexual responses that do not allow to enjoy the sexuality satisfactorily. The women can present disorders in the desire, arousal, orgasm or pain. Even though is frequent the coexistence of two or more dysfunctions.

Médico. Especialista en Ginecología y Obstetricia. Universidad Militar Nueva Granada. Bogotá. Colombia. Magíster en Sexología. Universidad de Alcalá de Henares. 
Objective: to determine the prevalence of female sexual dysfunction in a population from Eje Cafetero- Colombia.

Methods: a prospective, transverse, descriptive and applicative study was carried out; also, it was applied the Female Sexual Function Index (FSFI) in 20.268 women between 18 and 80 years old that took consultations in the main medical centers in the Eje Cafetero's cities (Armenia, Manizales y Pereira).

Results: regarding the different sexual dysfunction prevalence, in general terms, the most frequent were sexual desire disorder (34.9\%), followed by orgasm absence, lack of lubrication $(19.5 \%)$, arousal disorder $(20.2 \%)$, pain during sexual relationship $(8.4 \%)$. The women of reproductive age showed a prevalence of $27.4 \%$ in desire disorders, while in menopausal women the ranks showed prevalence of $42.9 \%$ in desire disorders. The sexual dysfunction was associated with less satisfaction in sexual and intimate relationship with emotional states and negative feelings $(p<0.001)$.

Conclusion: there is a significant proportion of women with sexual dysfunction in the Eje Cafetero-Colombia (34.94\%). Rev.cienc.biomed. 2016;7(1):25-33.

\section{KEYWORDS}

Sexual dysfunction; Epidemiology; Sexual habits.

\section{INTRODUCCIÓN}

La sexualidad es una construcción psicosocial que comprende aspectos biológicos, culturales, de relación y subjetivos, abarca todas las prácticas físicas y mentales realizadas con la finalidad de obtener placer sexual (consigo mismo o con otro), e integra la identidad personal y contribuye con la reproducción humana (1).

La sexualidad femenina posee una plasticidad erótica mayor que la masculina, debido a que soporta mayor presión de los estándares socioculturales de belleza y atractivo sexual (2); no obstante, es un aspecto central de la calidad de vida de la mujer, y es reflejo de su bienestar físico, psicológico y social (3).

La sexualidad humana es un fenómeno complejo y un aspecto central del ser humano que está presente a lo largo de toda su vida (4), abarca el sexo, las identidades y los roles de género, el erotismo, el placer, la intimidad, la orientación sexual y la reproducción. Se vive y se expresa a través de actitudes, conductas, creencias, deseos, fantasías, pensamientos, prácticas, relaciones interpersonales y valores (5). Además, está influida por la interacción de factores biológicos, culturales, económicos, espirituales, éticos, históricos, legales, políticos, psicológicos, religiosos y sociales, factores propios de cada mujer y que dotan de características individuales (6-8).
Las disfunciones sexuales se definen como la alteración de una o más etapas del ciclo de respuesta sexual (deseo, excitación, orgasmo y resolución), que le impide al individuo o a su pareja el disfrute de la actividad sexual de modo satisfactorio $(9,10)$, lo que genera un malestar personal significativo que puede tener impacto en la calidad de vida personal y/o en pareja. Estas se presentan en cualquier individuo, independientemente de la edad, el sexo, la raza, la religión, el estado civil, etc. Suelen ser muchas y diversas, las causas que sobrellevan a los trastornos de la respuesta sexual se pueden presentar en cualquier etapa de la vida sexual del individuo $(11,12)$. En la mujer la disfunción sexual implica una serie de conflictos, entre los que se encuentran la disminución del deseo sexual o una baja motivación, la reducción de la lubricación vaginal o de la excitación, la disminución de la capacidad para alcanzar el orgasmo y la presencia de dolor sexual (13). El objetivo es determinar la prevalencia de disfunción sexual femenina en una población del Eje Cafetero colombiano, y, analizar si existen diferencias entre las mujeres en etapa reproductiva y las climatéricas.

\section{MATERIALES Y MÉTODOS}

Se realizó un estudio tipo encuesta, prospectivo, trasversal, descriptivo y aplicativo, realizado en un grupo de mujeres del Eje Cafetero colombiano. Se aplicó el Índice de Función Sexual Femenino (IFSF) en entrevista personal, voluntaria, y en recinto privado a 20.268 
mujeres de 18 a 84 años de edad que aceptaron ser encuestadas voluntariamente, estas acudieron por primera vez a la consulta externa de ginecología y obstetricia en seis de los principales centros de salud de las ciudades del Eje Cafetero. Esta última es una región, geográfica, cultural, económica y ecológica de Colombia, localizada entre los departamentos de Caldas, Risaralda y Quindío y el noroccidente del departamento del Tolima. Cuenta con una población de, aproximadamente, 2.7 millones de habitantes, cuyas mujeres representan alrededor del 51\%.

El IFSF se complementó con datos sociodemográficos que se anexaron al formulario, como la edad, nivel académico, estado civil, ocupación, paridad, frecuencia promedio de relaciones sexuales, tabaquismo, ingesta de alcohol, método de anticoncepción, cirugía ginecológica o urológica, uso de terapia hormonal de reemplazo, antecedente de abuso sexual o violencia sexual en el matrimonio, recurrencia a la masturbación, condición espiritual, ocupación, área de origen e identidad sexual; todas estas actividades fueron realizadas por el grupo de enfermeras encuestantes, quienes también instruían a las mujeres en el autodiligenciamiento del IFSF, además de tallarlas y pesarlas para luego calcular el IMC. Al final del cuestionario cada mujer firmó el documento dando su consentimiento informado.

El estudio se realizó de forma prospectiva desde el 1 de julio de 2009 hasta el 30 junio de 2013, teniendo la precaución de buscar en la base de datos que no se repitiera la misma paciente, con el fin de evitar que participara varias veces de la encuesta. En Colombia no se cuenta, según la búsqueda bibliográfica realizada por el autor de este estudio, con escalas propias para la evaluación de la disfunción sexual femenina, sin embargo, existen trabajos americanos con herramientas válidas para evaluarla, como el Índice de Función Sexual Femenina (IFSF)/ Female Sexual Function Index (FSFI) de alta confiabilidad y fiables propiedades psicométricas en la evaluación de la función sexual femenina (14).

El IFSF se caracteriza por ser autoadministrado, simple y confiable para evaluar la fun- ción sexual femenina en un amplio rango de edad, ha demostrado altos niveles de confiabilidad, consistencia interna y propiedades psicométricas para la evaluación de la función sexual femenina (15). Este cuestionario consta de 19 preguntas que se agrupan en seis dominios: deseo (ítems $1-2$ ), excitación (ítems 3 - 6), lubricación (ítems $7-10$ ), orgasmo (ítems 11 - 13), satisfacción (ítems 14 - 16) y dolor (ítems 17 - 19). Cada pregunta tiene 5 o 6 opciones, asignándoles un puntaje que va de 0 a 5 . Los aspectos se evalúan con preguntas cuantitativas respecto al tema específico, cuya respuesta se gradúa según el siguiente puntaje: 0 ). Sin actividad sexual; 1 ). Casi nunca; 2 ). Menos de la mitad de las veces; 3 ). La mitad de las veces; 4). Más de la mitad de las veces; 5). Casi siempre (16). El puntaje de cada dominio se multiplica por un factor y el resultado final es la suma aritmética de los dominios, a mayor puntaje mejor sexualidad. Se considera alterado un aspecto con un puntaje igual o menor a 3 en cada ítem preguntado, excepto las preguntas referentes a dolor, en las cuales se consideró alterado un puntaje igual o mayor a 3 . El punto de corte para definir la disfunción sexual es un puntaje menor a 26.55 puntos (17).

Los criterios de inclusión fueron: mujeres que aceptaron participar en el estudio, mayores de 18 años, con pareja estable (por lo menos durante los últimos seis meses), con actividad sexual en los últimos tres meses y que acudieron por primera vez a la consulta externa de ginecología y obstetricia. Se excluyeron las mujeres menores de 18 años, las embarazadas y lactantes, las que no desearon participar, las que previamente presentaban disfunción sexual, con déficit mental que les impidiera entender o diligenciar el cuestionario, tercer trimestre del embarazo, primeras seis semanas postparto o las que presentaban alguna enfermedad que las incapacitara para tener vida sexual.

Se hizo un análisis de las características sociodemográficas de la población general, luego se realizó un análisis comparativo entre las características sociodemográficas de las mujeres en edad reproductiva (menores de 40 años) y climatéricas (mayores de 40 años). 
Los datos estadísticos fueron analizados con el software SAS (v9.2) e InfoStat versión 2012. Se realizaron análisis descriptivos univariados, bivariados, tanto con el IFSF ( $y$ sus dominios), como con las variables biosociodemográficas. En el estudio estadístico para variables cuantitativas se expresan los datos en rango, media y desviación estándar ( $\pm D E)$, mientras que las variables categóricas se expresaron como frecuencia numérica (n) y relativa (\%). Para la comparación de medias se utilizó el test de la T de Student. En el caso de no normalidad se expresaron las variables numéricas como mediana. Para determinar la relación entre el IFSF (y sus dominios) y las variables biosocio-demográficas cuantitativas se utilizó el coeficiente de correlación de Pearson (Spearman, en caso de que no se cumpliera la normalidad), y se aplicó análisis de varianza, cuando las variables biosocio-demográficas eran categóricas. Este análisis se realizó en general y por cada estamento, la significación estadística se consideró con una $\mathrm{p}<0.05$.

\section{RESULTADOS}

Se solicitó a 28.749 mujeres que diligenciaran la encuesta del IFSF bajo la orientación de las enfermeras de campo, capacitadas en su diligenciamiento, quienes acompañaron a cada una de las mujeres encuestadas. Se retiraron de la encuesta 5.142 (17.8\%) mujeres por sentirse incómodas con algunas preguntas, se eliminaron $2.136(7.4 \%)$ formularios por estar incompletos y 1.203 $(4.1 \%)$ no desearon participar; por lo tanto, el análisis final del estudio se realizó con 20.268 (70.4\%) formularios. El estudio fue aprobado por el Comité Ético Científico del servicio de salud correspondiente.

La Tabla No 1 muestra las características sociodemográficas de la población general y la Tabla No 2 muestra las características sociodemográficas de ambos grupos. En la población general el promedio de edad fue $36.9 \pm 8.4$ y de nivel educativo $11.4 \pm 4.8$ años (la mayoría de predominio urbano $87.0 \%$ ), la paridad fue $2.4 \pm 2.1$. El $5.9 \%$ eran fumadoras e ingerían alcohol el $31.5 \%$, el $5.0 \%$ eran menores de 19 años, el $24.8 \%$ eran postmenopáusicas y el $3.6 \%$ eran usuarias de terapia de remplazo hormonal.

\begin{tabular}{|c|c|}
\hline $\begin{array}{l}\text { TABLA No } 1 . \\
\text { CARACTERÍSTICAS SOCIODEI } \\
\text { DE LA POBLACIÓN GE }\end{array}$ & $\begin{array}{l}\text { OGRÁFICAS } \\
\text { ERAL }\end{array}$ \\
\hline Edad, $X \pm D E$ & $36.9 \pm 8.4$ \\
\hline Peso, $X \pm D E$ & $72.3 \pm 11.4$ \\
\hline Talla, $X \pm D E$ & $1.62 \pm 0.3$ \\
\hline $\mathrm{IMC}, \mathrm{X} \pm \mathrm{DE}$ & $27.6 \pm 4.8$ \\
\hline Nivel académico, $\mathrm{X} \pm \mathrm{DE}$ & $\begin{array}{l}11.4 \pm 4.8 \\
\text { años }\end{array}$ \\
\hline Paridad $X \pm D E$ & $2.4 \pm 2.1$ \\
\hline Unión libre,\% & 57.0 \\
\hline Anticoncepción hormonal oral,\% & 65.8 \\
\hline Fumadoras, $\%$ & 5.9 \\
\hline Ingesta alcohol, \% & 31.5 \\
\hline $\begin{array}{l}\text { Uso actual de terapia } \\
\text { hormonal, } \%\end{array}$ & 3.6 \\
\hline Menores de 18 años, $\%$ & 5.0 \\
\hline Postmenopáusicas,\% & 24.8 \\
\hline Amas de casa, \% & 42.1 \\
\hline Urbana, \% & 87.0 \\
\hline $\begin{array}{l}\text { Cirugía ginecológica / urológica, } \\
\%\end{array}$ & 6.1 \\
\hline Heterosexual, \% & 99.3 \\
\hline
\end{tabular}

\begin{tabular}{|c|c|c|}
\hline \multicolumn{3}{|c|}{$\begin{array}{c}\text { TABLA No } 2 . \\
\text { CARACTERÍSTICAS SOCIODEMOGRÁFICAS } \\
\text { DE LAS DOS POBLACIONES }\end{array}$} \\
\hline & \begin{tabular}{|c|} 
Etapa \\
reproductiva \\
\end{tabular} & $\begin{array}{c}\text { Etapa } \\
\text { climatérica }\end{array}$ \\
\hline Edad, $X \pm D E$ & $30.2 \pm 1.3$ & $46.1 \pm 2.5$ \\
\hline Peso, $X \pm D E$ & $60.8 \pm 2.2$ & $64.7 \pm 2.8$ \\
\hline Talla, $\mathrm{X} \pm \mathrm{DE}$ & $1.65 \pm 0.3$ & $1.61 \pm 0.4$ \\
\hline $\mathrm{IMC}, \mathrm{X} \pm \mathrm{DE}$ & $27.6 \pm 4.8$ & $29.5 \pm 4.1$ \\
\hline $\begin{array}{l}\text { Nivel académico, } \\
X \pm D E\end{array}$ & $\begin{array}{l}11.9 \pm 3.7 \\
\text { años }\end{array}$ & $\begin{array}{l}10.1 \pm 2.8 \\
\text { años }\end{array}$ \\
\hline Paridad $\mathrm{X} \pm \mathrm{DE}$ & $1.7 \pm 0.7$ & $3.8 \pm 0.7$ \\
\hline Unión libre,\% & 83.4 & 43.71 \\
\hline Fumadoras, \% & 7.9 & 4.8 \\
\hline Ingesta alcohol, \% & 37.2 & 17.4 \\
\hline $\begin{array}{l}\text { Menores de } 19 \\
\text { años,\% }\end{array}$ & 7.7 & - \\
\hline 19 a 39 años, \% & 92.2 & - \\
\hline $\begin{array}{l}\text { Transición } \\
\text { menopausia, \% }\end{array}$ & - & 10.3 \\
\hline Perimenopaúsicas,\% & - & 18.0 \\
\hline Postmenopáusicas,\% & - & 71.6 \\
\hline Amas de casa; \% & 37.1 & 58.7 \\
\hline Urbana,\% & 71.3 & 81.2 \\
\hline $\begin{array}{l}\text { Cirugía } \\
\text { ginecológica/ } \\
\text { urológica, \% }\end{array}$ & 4.5 & 7.4 \\
\hline Heterosexual,\% & 88.5 & 99.8 \\
\hline
\end{tabular}


En la población general, el estado civil más frecuente fue la unión libre $(57.0 \%)$. Lo más frecuente fueron los estudios secundarios (57.4\%); el $57.8 \%$ de las mujeres trabajaba y el $42.1 \%$ eran amas de casa. En el grupo de las mujeres climatéricas a 2.517 de ellas $(35.7 \%)$ se les había realizado histerectomía, de las cuales a 957 (13.5\%) se les realizó salpingooforectomía bilateral concomitante. El $58.7 \%$ presentaban enfermedades, las más frecuentes fueron: diabetes mellitus tipo 2 en 1.539 casos (37.1\%), hipotiroidismo en 1.026 casos (24.7\%), hipercolesterolemia en 969 casos $(23.3 \%)$ e hipertensión arterial en 609 casos (14.6\%).

En el 34.9\% (7.083) de la población general de mujeres encuestadas, con base en el puntaje obtenido en el IFSF, se presentó disfunción sexual en al menos uno de los aspectos analizados. De estas, 1.404 mujeres $(6.9 \%)$ presentaron alteración en dos aspectos $y, 2.181$ mujeres (10.7\%) en más de dos aspectos. El $4.713(23.2 \%)$ tenían dificultades en las relaciones sexuales, pero no en la masturbación y el 9.084 (44.8\%) no se masturbaba.

En este estudio, las mujeres en etapa reproductiva mostraron prevalencia del $27.4 \%$ de trastornos del deseo, $15.9 \%$ inhibición de la excitación, $13.5 \%$ falla en la lubricación, $11.8 \%$ ausencia de orgasmos, $10.8 \%$ falta de satisfacción sexual y $6.2 \%$ dispareunia, mientras que en las mujeres en etapa climatérica los rangos mostraron prevalencia del $42.9 \%$ de trastornos del deseo, $28.6 \%$ en inhibición de la excitación, el $25.7 \%$ falla en la lubricación, el $26.5 \%$ con ausencia de orgasmos, el $18.0 \%$ falta de satisfacción sexual y el $11.6 \%$ dispareunia.

En la población general el promedio en la frecuencia de relaciones sexuales fue de 9.6 veces por mes (rango de 1 a 27 veces mensuales). En este estudio, en la población general, de las 7.083 mujeres que presentaron disfunción sexual, el 4.9\% (351) habían reducido la frecuencia en el número de encuentros íntimos como resultado de la disfunción que presentaban.

El dominio más alterado fue el deseo sexual, presentando el menor de los puntajes
(3.6士1.2) y una alteración en 7.077 mujeres (34.9\%). El segundo aspecto más alterado fue la ausencia de orgasmo o marcada infrecuencia en el orgasmo, con un puntaje $3.9 \pm 1.5$ que se presentó en 5.781 mujeres (28.5\%). La falta de lubricación se presentó en el $19.5 \%$ de las mujeres y su puntaje fue $5.1 \pm 1.2$, la excitación estuvo alterada en el $20.2 \%$ de las mujeres y el dolor en la relación sexual estuvo presente en el $8.4 \%$, lo que muestra que estos dos últimos dominios fueron los que presentaron el mayor puntaje ( $4.8 \pm 1.8$ y $5.1 \pm 1.5$ respectivamente). La presencia de insatisfacción sexual fue referida por el $8.4 \%$ de las mujeres, la que mostró un puntaje de $4.8 \pm 2.4$ (Tabla No 3 ).

\begin{tabular}{|c|c|c|c|}
\hline Índice de & $\begin{array}{r}\text { TABLA } \\
\text { Función Sex } \\
\text { iujeres del }\end{array}$ & $\begin{array}{l}\text { No } 3 . \\
\text { ual Femen } \\
\text { je Cafeter }\end{array}$ & no de las \\
\hline Dominios & $\begin{array}{l}\text { Reproducti- } \\
\text { vas }(\%)\end{array}$ & $\begin{array}{l}\text { Climatéri- } \\
\text { cas }(\%)\end{array}$ & $\begin{array}{c}\text { Población } \\
\text { general } \\
(\%)\end{array}$ \\
\hline Deseo & 27.4 & 42.9 & 34.9 \\
\hline Excitación & 15.9 & 28.6 & 20.2 \\
\hline $\begin{array}{l}\text { Lubrica- } \\
\text { ción }\end{array}$ & 13.5 & 25.7 & 19.5 \\
\hline Orgasmo & 11.8 & 26.5 & 28.5 \\
\hline $\begin{array}{l}\text { Satisfac- } \\
\text { ción }\end{array}$ & 90.2 & 81.9 & 86.2 \\
\hline Dolor & 6.2 & 11.6 & 8.4 \\
\hline
\end{tabular}

A la pregunta sobre la importancia del sexo en la relación de pareja, 18.603 mujeres, que corresponden al $91.7 \%$ de las encuestadas, consideraron la actividad sexual como un aspecto fundamental en la relación con el vínculo (independientemente del estatus marital), lo cual muestra que la sexualidad tiene un impacto importante en el contexto socio-familiar y de pareja en la mujer del Eje Cafetero.

La mayoría de las mujeres del estudio se encontraban en edad fértil, siendo estas 13.221 $(65.2 \%)$, mostrando un promedio del IFSF de $29.4 \pm 4.8$ puntos, mientras que el $24.8 \%$ estaban en etapa climatérica, con un puntaje de $24.3 \pm 4.5$ puntos. El grupo de las usuarias de terapia de remplazo hormonal arrojaron en el IFSF un puntaje de 25.5 29.3 .

En cuanto al origen urbano o rural se encontró un $24.6 \%$ de disfunción sexual en muje- 
res del área rural que obtienen un promedio de índice menor (27.3 \pm 5.1$)$, mientras que aquellas mujeres del área urbana obtienen

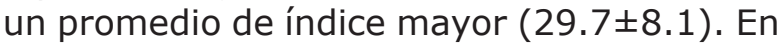
el grupo de mujeres en edad reproductiva, el método anticonceptivo más utilizado es el hormonal oral en 8.712 (65.8\%), grupo que obtiene un promedio de $28.5 \pm 4.2$ puntos en el IFSF, seguido por el $29.5 \%$ de usuarias del implante subdérmico, con promedio de $29.1 \pm 6.3$ puntos en el IFSF, mientras que $4029(4.5 \%)$ no utilizaban ningún método anticonceptivo, a pesar de no tener intención reproductiva y obtuvieron $26.9 \pm 6.9$ puntos promedio en el IFSF.

En la población general, el antecedente de abuso sexual, maltrato infantil y/u otro tipo de agresión estuvo presente en el $16.9 \%$ de las mujeres encuestadas, las que obtuvieron

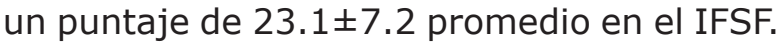
Asimismo, el grupo de mujeres que obtuvo un IFSF menor de 26.5 puntos el $78.8 \%$ de ellas relacionaban el tipo de trastorno sexual con los problemas sexuales o de salud de la pareja (disfunción eréctil en un 3.3\%, eyaculación precoz en un $3.9 \%$ e hiperplasia prostática en un $2.4 \%$, logrando puntajes promedios en el IFSF de $16.3 \pm 8.4,25.3 \pm 8.7$ y $21.7 \pm 9.6$ puntos, respectivamente).

\section{DISCUSIÓN}

La prevalencia de disfunción sexual femenina varía según el instrumento utilizado en cada estudio, lo que identifica diferentes subgrupos, por lo tanto, el instrumento de investigación afecta la prevalencia (18). En los últimos años han comenzado a aparecer en la literatura médica, los primeros trabajos de investigación y revisión sobre la función sexual femenina y sus disfunciones $(19,20)$. No en vano, el más citado y que ha servido como patrón de referencia en el estudio de la sexualidad femenina, es el cuestionario del IFSF (21).

Las disfunciones sexuales son más frecuentes en mujeres $(43.0 \%)$ que en hombres $(31.0 \%)$, con el agravante de que el trastorno es mucho más complejo y multifactorial en la mujer (22). El grupo de consenso internacional calculó que la disfunción sexual femenina afecta a más del $20 \%$ de las mujeres
(10). En esta población de estudio, más de la tercera parte de las mujeres presentaban alguna disfunción sexual, sobrepasando las cifras de la mayoría de los estudios citados. Por lo tanto, habría que incidir en la idoneidad de la consulta médica interrogando a las mujeres acerca de estos trastornos, y alertar a la población de profesionales de atención primaria para que tengan en cuenta la salud sexual de la mujer.

El estudio global de actitudes y comportamientos sexuales (EGACS), una encuesta realizada (entre 2001 y 2002 ) a 27.500 personas: hombres (13.618) y mujeres (13.882) entre los 40 y 80 años de edad, en 29 países diferentes, incluidos 10.000 hombres y mujeres de 8 países europeos (Alemania, Austria, Bélgica, España, Francia, Italia, Reino Unido y Suecia) (23), aporta información sobre la actividad sexual y actitudes frente a la misma, evidenciándose la alta frecuencia de problemas sexuales relacionados fundamentalmente con la edad y con la presencia de enfermedades. En este estudio el $43.0 \%$ de los hombres y el $49.0 \%$ de las mujeres informaron, por lo menos, un trastorno sexual, estas últimas acusaban disfunción del tipo falta de interés sexual y la incapacidad para alcanzar el orgasmo. En la población española se entrevistaron a 750 mujeres, y los problemas sexuales más comunes descritos fueron la falta de interés sexual en un $36.0 \%$, la incapacidad de alcanzar el orgasmo en un $27.8 \%$ y las relaciones sexuales no placenteras en un $25.1 \%$ (24).

En este estudio las mujeres con menor actividad sexual tenían significativamente más edad, un porcentaje menor tenía pareja estable con alguna disfunción sexual y el resto eran postmenopáusicas, por lo que la prevalencia de disfunción sexual era mayor y el puntaje total del IFSF tendía a ser menor de $26.55(p<0.0007)$. La prevalencia de disfunción sexual femenina reportada en este estudio se encuentra dentro del rango señalado en estudios previos (25).

El estudio de prevalencia de problemas sexuales femeninos asociados con angustia y determinantes en la búsqueda de tratamiento (PRESIDE, por sus siglas en inglés) (6) valoró la prevalencia de los problemas 
sexuales y el malestar derivado de las relaciones sexuales, incluyó 31.581 mujeres estadounidenses de 18 años o mayores (18 y 102) y utilizó cuestionarios validados para evaluar la función sexual y medir la angustia. El problema más común fue la falta de deseo, con una prevalencia ajustada a la edad del $37.7 \%$. La prevalencia de la disminución del deseo y angustia fue del $8.9 \%$ en mujeres entre los 18 y 44 años, del $12.3 \%$ en mujeres entre los 45 y 64 años y del $7.4 \%$ en mujeres mayores de 65 años. Al ajustar el perfil de edad en las mujeres estadounidenses, la prevalencia de la disminución del deseo asociada al malestar fue del $9.5 \%$. Las cifras de alteraciones en el orgasmo señalan que un $10 \%$ de las mujeres casadas no lo experimentan $(26,27)$, otros números van desde el $11.6 \%$ (28) al $37 \%$ de las mujeres (16); sin embargo, estudios más recientes oscilan entre rangos del $20 \%$ y $50 \%$ (29).

La prevalencia de los trastornos sexuales femeninos, diagnosticables, es del $12.0 \%$. En EE.UU. el $43.0 \%$ de las mujeres de 18 a 59 años tienen trastornos sexuales y aproximadamente el $40 \%$ de las mujeres experimentan algún tipo de problema sexual en el transcurso de su vida $(6,21)$. No obstante, los índices de prevalencia de disfunción sexual femenina varían entre 40 y $53.8 \%$ $(8,30,31)$. En este estudio un $34.91 \%$ de las mujeres encuestadas presentaban trastornos sexuales.

La disfunción sexual más prevalente en las mujeres de todas las edades es la disminución del deseo sexual: 51\% (deseo sexual hipoactivo y la aversión sexual), $(32,33)$ anteriormente denominado trastorno del deseo sexual hipoactivo en la cuarta edición del DSM, texto revisado (30) (DSM-IV-TR) y trastorno del interés y la excitación sexual femenina en la quinta edición del DSM (DSM5) (34). En estos resultados se demuestra nuevamente esta prevalencia, puesto que la disfunción sexual más frecuente, tanto en las mujeres en edad reproductiva como en las menopaúsicas, es la disminución del deseo sexual; las alteraciones del orgasmo representan el $18.5 \%$, porcentajes menores a los registrados en otros estudios, que destacan el $24.0 \%$ (13).
Algunos autores describen que el $20.0 \%$ de las mujeres manifiestan dificultades en la lubricación, el $20.0 \%$ refieren no tener sexo placentero, y más del $40.0 \%$ acusan deseo infrecuente $y / o$ que no disfrutan el sexo placenteramente (35), lo que no coincide con la población general de este estudio que refleja cifras inferiores, ya que las dificultades en la lubricación representan el $14.8 \%$, mientras que la satisfacción es del $88.2 \%$.

Las cifras de trastorno de dolor durante la práctica sexual en mujeres en edad reproductiva muestran que la prevalencia de dispareunia se encuentra en el rango de 10.0 a $15.0 \%(21,36)$, cosa que no concuerda con las cifras reportadas por la población en edad reproductiva, ya que se caracterizan cifras inferiores $(3.9 \%)$, y $5.7 \%$ en las climatéricas, con un $8.4 \%$ para la población general del estudio.

En este estudio se encontró una prevalencia de disfunciones sexuales, mayor en las mujeres climatéricas que en aquellas en edad reproductiva, pero la prevalencia de satisfacción en estas últimas fue inferior a las de las climatéricas, lo que puede estar relacionado con varios factores: uno es el aspecto cultural, ya que por tratarse de mujeres mayores, interpretan la actividad coital como un marcador de buena sexualidad, independientemente de las variables del ciclo de respuesta sexual; en segundo lugar, las mujeres jóvenes demandan un mejor encuentro íntimo, con exigencias de sus respectivos orgasmos y satisfacción general.

La disfunción del deseo sexual reflejado en el estudio de estas mujeres hizo que se mostraran insatisfechas con su vida sexual $(p<0.001)$, con su relación $(p<0.001)$, manifiestan más emociones negativas $(p<0.001)$ y peores relaciones íntimas. Se pudo observar en este estudio que mujeres que experimentaron violencia sexual en diferentes etapas de sus vidas fueron más proclives a reportar bajos puntajes en el IFSF.

La violencia sexual contra las mujeres dentro del matrimonio o dentro de las uniones consensuales no fueron un evento poco común en este estudio, con una cifra porcentual del $5.3 \%$. Las mujeres participantes del estudio 
han sido forzadas a tener relaciones sexuales con su pareja cuando ellas no lo deseaban. Las mujeres declararon su condición de pareja estable, ya sea por estar casadas, en unión libre o por tener una relación de pareja conviviente; el $99.3 \%$ de ellas se consideró heterosexual.

El presente estudio tiene algunas limitaciones: primero, es importante señalar que en esta investigación solamente participaron mujeres que asistieron por primera vez a consulta externa, lo que puede limitar algunos aspectos de la educación sexual especializada; segundo, la selección de las mujeres se realizó directamente en la consulta de ginecología y obstetricia; tercero, en este estudio se encontró que las mujeres en edad reproductiva representaron más de las dos terceras partes de la población general; cuarto, no se consideró el nivel socioeconómico de las mujeres encuestadas, lo que podría influir en algunas de las variables asociadas como el nivel académico.

A pesar de estas limitaciones, se puede afirmar que se encontró una elevada prevalencia de disfunciones sexuales en la muestra estudiada de mujeres del Eje Cafetero. El impacto de algunas variables de salud y socioculturales sobre el riesgo de tener disfunción sexual se observó en edades superiores a los 45 años, paridad mayor de dos hijos, el estado postmenopáusico, las cirugías pélvicas, el antecedente de abuso sexual y la histerectomía. La asistencia o pertenencia a un grupo religioso en particular fue un factor que se puede señalar como posiblemente protector ante la presencia de una pareja estable.

A fin de contribuir con el bienestar sexual de las mujeres colombianas, los programas de educación y asesoramiento sexual deben fomentarse desde edades tempranas; por lo tanto, los médicos, y en especial los ginecólogos, deben enseñar a perder el temor a la consulta, y a su vez, se debe aprender a preguntar sobre los trastornos sexuales, puesto que la mujer con disfunción sexual tiene derecho a ser estudiada. Se deben diseñar estudios específicos para evaluar el impacto que las disfunciones sexuales puedan acarrear en la vida de las mujeres del país.

\section{CONCLUSIÓN}

En la tercera parte de las mujeres estudiadas en el Eje Cafetero, se observó disfunción sexual, por ello es un problema frecuente. No obstante fue baja la existencia de insatisfacción sexual.

CONFLICTO DE INTERESES: ninguno que declarar.

FINANCIACIÓN: recursos propios de los autores.

AGRADECIMIENTOS: el autor agradece a las voluntarias que participaron en el estudio.

\section{REFERENCIAS BIBLIOGRÁFICAS}

1. Villela WV, Arilha M. Sexualidade, Gênero e direitos sexuais e reprodutivos. In: Berquo E, editor. Sexo \& Vida- Panorama da Saude Reprodutova no Brasil. Campinas: Editora Unicamp; 2003:95-150.

2. Woertman L, Van Den Brink F. Body Image and female sexual functioning and behavior: A Review. J Sex Res. 2012;49(2-3):184-211.

3. Ojanlatva A, Makinen J, Helenius H, Korkeila K, Sundell J, Rautava P. Sexual activity and perceived health among finnish middle-aged women. Health Qual Life Outcomes 2006; 4: 29.

4. Blumel J, Araya H, Riquelme R, Castro G, Sánchez F, Gramegna G. Prevalencia de los trastornos de la sexualidad en mujeres climatéricas. Influencia de la menopausia y de la terapia de reemplazo hormonal. Rev Méd Chile 2002; 130: 131-8.

5. Gagnon J. Uma interpretação do desejo: ensaios sobre o estudo da sexualidade: Editora Garamond; 2006.

6. Shifren JL, Monz BU, Russo PA, Segreti A, Johannes CB. Sexual problems and distress in United States women: prevalence and correlates. Obstet Gynecol 2008; 112:970-8.

7. Basson R, Brotto LA, Laan E, Redmond G, Utian WH. Assessment and management of women's sexual dysfunctions: problematic desire and arousal. J SexMed 2005; 2: 291-300.

8. Paul RN, Kleeman SD, Karram MM. Female sexual dysfunction: principles of diagnosis and therapy. Obstet Gynecol Surv. 2005;60(3):196-205. 
9. Conaglen HM, Conaglen JV. Drug-induced sexual dysfunction in men and women. Austr Prescr. 2013;36:42-5.

10. Basson R, Berman J, Burnett A, Derogatis L, Ferguson D, Fourcroy J, et al. Report of the international consensus development conference on female sexual dysfunction: definitions and classifications. J Urol 2000;163:888-93.

11. Sasson A, Leiblum S. Revised Definitions of women's sexual dysfunctions. Journal of Sexual medicine 2004;1:40-8.

12. Kaplan, H. Disorders of sexual desire and other new concepts and techniques in sex therapy. New York: Simon \& Schuster. (1979).

13. American Psychiatric Association. Diagnostic and statistical manual of mental dDisorders. DSM-IV. Washington, DC: American Psychiatric Association; 2000.

14. Osborn M, Hawton K, Gath D. Sexual dysfunction among middle aged women in the community. Br Med J (Clin Res Ed) 1988;296(6627):959-962.

15. Althof SE, Rosen RC, DeRogatis L, Corty E, Quirk F, Symonds T. Outcome measurement in female sexual dysfunction clinical trials: review and recommendations. J Sex \& Marital Ther. 2005 Mar-Apr;31(2):153-166.

16. Blümel J, Binfa L, Cataldo A, Carrasco V, Izaguirre L, Sarrá C. Índice de función sexual femenina: un test para evaluar la sexualidad de la mujer. Rev Chil Obstet Ginecol. 2004;69(2):118-125.

16. Rosen R, Braown C, Heiman J. Female Sexual Funtion Index (FSFI): a multidimensional self-report instrument for the assessment of female sexual function. J. Sex Marital Ther. 2000;26:191-208.

17. Cindy M. Validation of the Female Sexual Function, Index (FSFI) in Women with Female Orgasmic Disorder and in Women with Hypoactive, Sexual Desire Disorder, MESTON. Journal of Sex \& Marital Therapy 2003; 29: 39-46.

18. Nusbaum MR, Gamble G, Skinner B, Heiman J. The high prevalence of sexual concern among women seeking routine gynecological care. J Fam Pract 2000; 49(3): 229-32.

19. Genazzani AR, Nicolucci a, Campagnoli C, Crosignanip, Nappi C, Serra GB ET AL. Assessment of the QoL in Italian menopausal women: comparison between HRT users and non-users. Maturitas 2002; 42: 267-80.

20. Abdo CHN, Oliveira WM, Moreira ED JR, Fittipaldi JAS. Prevalence of sexual dysfunctions and correlated conditions in a sample of Brazilian women- results of the Brazilia study in sexual behaviour (BSSB). Int J Impot Res 2004; 16: 160-6.

21. Sexual problems common among women and men. Women's Health Weekly; 1999;17:16-18.

22. Vardi Y, Gruenwald I, Sprecher E, Gertman I, Yarnitsky D. Normative values for female genital sensation. Urology 2000; 56:1035- 40.

23. Dennerstein $L_{1}$ : Third Annual female sexual function forum: New perspectives in the Management of Female Sexual Dysfunction. www.medscape.com/WomensHealth/journal/2000/v05.n06/whl1205.denn/wh1205.denn.html.

24. Laumann EO, Nicolosi A, Glasser DB, Paik A, Gingell C, Moreira E, et al. Sexual problems among women and men aged 40-80 y: prevalence and correlates identified in the Global Study of Sexual Attitudes and Behaviors. Int J Impot Res. 2005;17:39-57.

25. Hayes RD, Dennerstein L, Bennett CM, Fairley ChK. What is the "true" prevalence of female sexual dysfunctions and does the way we assess conditions have an impact? J Sex Med. 2008; 5: 777-87.

26. Moreira Jr ED, Glasser DB, Gingell C. Sexual activity, sexual dysfunction and associated help-seeking behaviours in middleaged and older adults in Spain: a population survey. World J Urol. 2005;23:422-9.

27. Chesser $\mathrm{E}$. The sexual marital and family relationships of the english woman. Londres: Hutchinson's Medical Publications; 1956.

28. Kinsey AC, Pomeroy WB, Martín CE, Gebhard P. Sexual behaviour in the human female. Filadelfia: W.B. Saunders Co; 1953.

29. Hite S. El informe Hite. Barcelona: Plaza y Janés; 1976.

30. Laumann EO, Paik A, Rosen RC. Sexual dysfunction in the United States. Prevalence and predictors. JAMA 1999; 281: 537-44.

31. Oksuz E, Malhan S. Prevalence and risk factors for female sexual dysfunction in Turkish women. J Urol.2006 Feb;175(2):654-8.

32. Ponholzer A, Roehlich M, Racz U, Temml C, Madersbacher S. Female sexual dysfunction in a healthy Austrian cohort: prevalence and risk factors. Eur Urol. 2005;47(3):366-74.

33. Basson, R. Clinical practice. Sexual desire and arousal disorders in women. N Engl J Med.2006;354:1497.

34. American Psychiatric Association, American Psychiatric Association, DSM-5 Task Force. Diagnostic and statistical manual of mental disorders: DSM-5. 5th ed. Washington, DC: American Psychiatric Association; 2013.

35. Weijmar W, Basson R, Binik Y, Eschenbach D, Wesselmann U, Van Lankveld J. Women's Sexual Pain and its Management. J Sex Med 2005;2:301-316.

36. West SL, Viniloor LC, Zolnoun D. A systematic review of the literature on female sexual dysfunction prevalence and predictors. Ann Rev Sex Res. 2004;15: 40-172. 